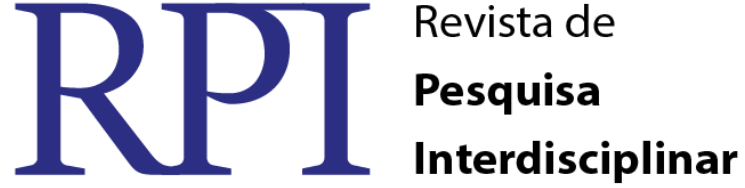

\section{EDUCACIONALIZACIÓN EN LA VIDA COTIDIANA Y SEXUALIDAD EL CASO DE LA LEY DE MATRIMONIO IGUALITARIO EN LA REPÚBLICA ARGENTINA.}

\author{
Néstor Pievi ${ }^{1}$ - UNSAM
}

\begin{abstract}
RESUMO
La elaboración de esta presentación responde a la necesidad de dar luz a preguntas relacionadas con dos cuestiones centrales que se entrelazan: una ha sido el anclaje y objetivación del objeto cultural matrimonio igualitario en la sociedad argentina, teniendo en cuenta la propuesta del Estado en el desarrollo de acciones cívicas que se orientan hacia la construcción de una sociedad inclusiva que garantice la igualdad de derechos a todos los ciudadanos con reconocimiento de la diversidad y la otra, el proceso de apropiación de este objeto cultural por parte de los sujetos como constituyente del aprendizaje en la vida cotidiana. Esta problemática nos lleva a preguntarnos sobre los procesos de anclaje, objetivación, dominación y apropiación de los significados socialmente compartidos, y como tal su relación con el proceso de educacionalización en la vida cotidiana.
\end{abstract}

Palabras claves: inclusion sociales; represetacion sociale.

\section{EDUCATIONALIZATION IN EVERYDAY LIFE AND SEXUALITY THE CASE OF THE LAW OF EQUALITY MARRIAGE IN THE ARGENTINE REPUBLIC.}

\begin{abstract}
The elaboration of this presentation responds to the need to shed light on questions related to two central issues that are intertwined: one has been the anchoring and objectification of the cultural object equal marriage in Argentine society, taking into account the state's proposal in development Of civic actions that are oriented towards the construction of an inclusive society that guarantees equal rights to all citizens with recognition of diversity and the other, the process of appropriation of this cultural object by the subjects as a constituent of learning in Everyday life. This problem leads us to ask about the processes of anchoring, objectification, domination and appropriation of socially shared meanings, and as such their relation to the process of education in everyday life.
\end{abstract}

Keywords: inclusive society. Social representations.

\section{INTROCIÓN}

Luego de seis años de sancionada la ley de Matrimonio Igualitario ${ }^{2}$, las expresiones discriminadoras homofóbicas siguen estando presentes en diferentes situaciones de la vida

\footnotetext{
${ }^{1}$ Magíster en Psicología Cognitiva y Aprendizaje- FLACSO y Universidad Autónoma de Madrid. Docente en enseñanza media y superior. Profesor en las cátedras: Psicología del desarrollo y aprendizaje, Psicología Educacional, Didáctica General y Trabajo de Campo en el Instituto Superior del Prof. Joaquín V. González; Psicología de la Educación en la UNSAM.

${ }^{2}$ La ley de Matrimonio Igualitario se sancionó el 15 de julio de 2010
} 
cotidiana argentina ${ }^{3}$, en las prácticas discursivas de los diferentes grupos sociales El reconocimiento de la homosexualidad como una variante de la orientación sexual humana y no un trastorno o una enfermedad por parte de la American Psychological Association y la American Psychiatric Association, ${ }^{4}$ no ha implicado la apropiación por parte de los diferentes actores sociales de esta posición, existiendo grandes distancias entre el discurso de la cienciay del Estado en nuestro caso- con el discurso del sentido común.

El aprendizaje en la vida cotidiana se da siempre desde nuestra inserción en el mundo, en relación con un otro, en diferentes contextos y situaciones, permitiéndonos la construcción de significaciones entorno a diferentes objetos culturales. El aprendizaje informal se desarrolla en las actividades de la vida cotidiana relacionadas con la familia, los amigos, el trabajo, etc., no está estructurado en objetivos didácticos, duración ni soporte y no es su meta llegar a una certificación. Este proceso tiene a su vez un carácter selectivo, donde se priorizan, se privilegian unas acciones por sobre otras en la trama intersubjetiva, donde entra en juego una polifonía de voces (Bajtin, 1981 ). Diferentes autores (Lave y Wenger, 1991; Rogoff, 1990, 1998) han considerado a la intersubjetividad como un proceso complejo en el que el entendimiento compartido, teniendo en cuenta que la co-construcción de significados se desarrolla en un marco de significados y prácticas culturales, socio-culturalmente situadas.

Según Foucault (2002), existe una íntima relación entre procesos y momentos históricos, en la producción de discurso, relación donde saber y poder van tejiendo una trama que constituye las visiones y divisiones del mundo. Visión y división del mundo que permite la construcción- a decir de Bourdieu- de arbitrarios culturales. Estos arbitrarios culturales se construyen entorno a objetos culturales.

En esta línea, la sanción de la Ley de Matrimonio Igualitario en Argentina se constituye en una problemática social entorno a un objeto cultural: el matrimonio homosexual. Tanto la campaña de divulgación masiva sobre la ley de Matrimonio Igualitario en la República Argentina como la sanción de la ley se presentan como un momento de inflexión en la historia social pero a su vez ha posibilitado la objetivación, institucionalización y legitimación de acciones identificadas con zonas "no visibilizadas" de la sociedad.

\footnotetext{
${ }^{3}$ Informe realizado por el ODRT. El Observatorio de la Discriminación en Radio y Televisión es un espacio de cooperación institucional conformado por la Autoridad de Servicios de Comunicación Audiovisual(AFSCA), el Instituto Nacional contra la Discriminación, la Xenofobia y el Racismo (INADI) y el Consejo Nacional de las Mujeres (CNM). Este proyecto de trabajo conjunto surgió a partir de la propuesta №208 del Plan Nacional contra la Discriminación, aprobado mediante el Decreto $\mathrm{N}^{\circ} 1086 / 05$, dictado en virtud de compromisos internacionales asumidos por nuestro país.

${ }^{4}$ Más de 35 años de investigación científica objetiva y bien diseñada han demostrado que la homosexualidad, en sí misma, no se asocia con trastornos mentales ni problemas emocionales

American Psychological Association: http://www.apa.org/centrodeapoyo/sexual.aspx
}

RPI Revista de Pesquisa Interdisciplinar, Cajazeiras, v. 1, Ed. Especial, 30-40, set/dez. de 2016. 
Podemos decir entonces que el Matrimonio Igualitario se constituyó en un objeto social donde el debate, la difusión de información, las confrontaciones en los medios gráficos y televisivos, fueron parte de un proceso de educacionalización en la vida cotidiana. Diferentes argumentaciones de parte de los representantes de los diversos sectores de la cultura argentina se manifestaron a favor o en contra del matrimonio entre personas del mismo sexo.

Los objetos culturales como el matrimonio igualitario se elaboran por medio de acciones comunicativas, mediadas y situadas, no son directamente accesibles a la cognición sino que los sujetos podemos pensar en objetos porque son presentados por medio de signos.

Desde esta perspectiva, la comprensión del lenguaje deviene un punto central para el estudio del aprendizaje en la vida cotidiana.

El matrimonio igualitario se constituye en el objeto social y cultural de esta presentación alrededor del cual se ha construido un conjunto de significaciones en la educación informal. En el proceso de construcción social de este arbitrario el lugar de pedagogización, poder y autoridad del Estado, como precursor de la Ley de Matrimonio Igualitario en Argentina, así como su intencionalidad, cobra un rol preponderante a la hora de explorar las voces compartidas por los diferentes actores sociales.

Las políticas de derechos humanos han atravesado el discurso del gobierno argentino en ejercicio de sus funciones durante la sanción de la ley de Matrimonio Igualitario. Las diferentes campañas del INADI $^{5}$ dan cuenta de ello. No obstante ello, las acciones de no aceptación del otro diferente, forman parte de la acciones que llevan adelante los sujetos en la vida cotidiana, sea este otro diferente en su elección sexual, género, cultura, origen social, nacionalidad, etc.

Parece paradojal que en un mundo intercultural, heterogéneo, caracterizado por la inmediatez de las comunicaciones y el desplazamiento de comunidades de unos países hacia otros, el respeto por el otro diferente parece seguir siendo una cuestión compleja que atraviesa a todos los espacios sociales.

En este marco, desde el Gobierno Nacional Argentino se llevaron a cabo diferentes propuestas que han puesto su énfasis en el respecto por los derechos de las minorías. Existió de parte del Gobierno Nacional una clara intencionalidad de construcción de una subjetividad social que se enmarca en la integración de zonas no visibilizadas de significados y sentidos.

\footnotetext{
${ }^{5}$ El Instituto Nacional contra la Discriminación, la Xenofobia y el Racismo (INADI), es un organismo descentralizado, creado mediante la ley Nro 24.515, en el año 1995, y comenzó sus tareas en el año 1997. Desde el mes de marzo del 2005, por decreto presidencial Nro 184, se lo ubicó en la órbita del Ministerio de Justicia y de Derechos Humanos de la Nación Argentina.

RPI Revista de Pesquisa Interdisciplinar, Cajazeiras, v. 1, Ed. Especial, 30-40, set/dez. de 2016.
} 
En esta línea, la Ley de Matrimonio Igualitario ${ }^{6}$ ha posibilitado el acceso a derechos que antes eran reservados a una parte de la comunidad, es decir constituye una alternativa para posibilitar la inclusión de una minoría- los homosexuales- en las prácticas sociales de una mayoría. A su vez esta inserción de las minorías llevó a la visibilización de zonas de significados que antes estaban reservadas para un grupo en particular. La ley de matrimonio igualitario permitió la visibilización de un colectivo social cuyas prácticas discursivas pertenecían a zonas marginales de significación (Berger y Luckman, 1968).

Por medio de diferentes mediadores sociales y semióticos el Estado Nacional instaló al Matrimonio Igualitario como objeto de debate, conocimiento y de construcción social enraizado como tal en valores, experiencias, vivencias, creencias, tradiciones, normas que forman parte de la realidad cotidiana de los sujetos. Vemos en este caso cómo los productos culturales y las experiencias subjetivas e intersubjetivas se entrelazan cuando la gente cambia su concepción de la vida social, de las personas, de los derechos y actúa de acuerdo a ello, involucrándose de diferentes maneras con la dinámica del cambio social y cultural. Este proceso de inserción en el devenir cívico es un proceso de aprendizaje que se da en la vida cotidiana. Las prácticas discursivas cobran un rol fundamental en la comprensión tanto sustancial como funcional de este proceso.

Estas prácticas posibilitan la intersubjetividad y como tal la construcción de zonas de significados compartidas por los grupos sociales. Estas zonas de significados compartidos permiten, la construcción de núcleos condensadores de significados que son difundidos y compartidos en el grupo social, transmitidos en la vida cotidiana, en las acciones comunicativas. Estos núcleos constituidos por valores, creencias, normas, tradiciones, son los que permiten la elaboración de significaciones socialmente compartidas que responden a diferentes posicionamientos.

La exploración de los sentidos y significados -dominados y apropiados en el proceso semiótico de aprendizaje informal, en la divulgación, debate y sanción de la ley de matrimonio igualitario en la República Argentina- permitiría la comprensión de los procesos de educacionalización en la vida cotidiana y como tal la interpretación de los sentidos y significados elaboradas entorno al objeto cultural, estableciendo la relación entre el discurso oficial y las prácticas discursivas compartidas por la población estudiada.

\footnotetext{
${ }^{6}$ La ley de Matrimonio Igualitario se sancionó el 15/07/2010, y a puesto en debate la cuestión del matrimonio entre personas del mismo sexo en la República Argentina

RPI Revista de Pesquisa Interdisciplinar, Cajazeiras, v. 1, Ed. Especial, 30-40, set/dez. de 2016.
} 
En el estudio del objeto cultural matrimonio igualitario tres ejes se entrecruzan: matrimonio, homosexualidad y derechos humanos. Estos tres ejes a su vez encuentran en la sexualidad su punto de interacción.

Al respecto, la toma en cuenta de la perspectiva de Foucault en la Historia de la sexualidad nos permite adentrarnos en la sociogénesis de los procesos representacionales. Quisiera por ello retomar algunas consideraciones que creo valiosas para comprender el sustrato que forma parte de las acciones antes citadas.

En la propuesta foucaultiana aparece una vinculación estrecha entre sexo, saber y poder. Podríamos considerar que fue necesario en el siglo XIX la construcción de una cierta subjetividad social alrededor de, a decir de Bourdieu, un "arbitrario cultural", un arbitrario cultural que de alguna forma posibilitaría la consecución de unas metas relacionadas con la constitución de los estados nación modernos en occidente. En particular, la distribución del trabajo, la necesidad de concentrar las energías en la producción económica y en la reproducción social, lleva a la elaboración de un discurso sobre el sexo tipificándolo (Berger y Luckmann, 1968). En contraposición a otros momentos de la historia social e intelectual, en el siglo XIX se habla de sexo buscando la legitimación de un arbitrario cultural. La mejor forma de legitimación de dicho arbitrario fue la institución civil en occidente del matrimonio heterosexual, monogámico, donde el sexo sólo tenía sentido en ese contexto. La sexualidad era adjudicada a los adultos (los niños no entraban en esta consideración) y en especial para una función: la reproducción. Era necesario distanciar a la sexualidad de cualquier acción que no se llevara en el seno del matrimonio.

La relación entre poder, saber y sexualidad se ha ido transformando según las diferentes épocas, contextos sociales, culturales, y decisiones gubernamentales. Los diferentes mediadores cobran un rol preponderante al momento de considerar las significaciones que los sujetos han co-construídos entorno a la institución matrimonio.

En el caso particular de la Argentina es necesario identificar al Estado Nacional como generador de la propuesta de modificación del Código Cívil en pos de la sanción de la ley de Matrimonio Igualitario.

Junto al Estado Nacional, los medios de comunicación social se ocuparon de la difusión y transmisión de las significaciones que se habían elaborado en torno al objeto social: matrimonio igualitario. La acción del Estado cobra una dimensión específica en este proceso de educacionalización como agente que posibilita o restringe el acceso a determinados derechos civiles, es el Estado quien otorga legitimidad a un posicionamiento grupal frente a 
un hecho cívico. Desde esta perspectiva, los derechos son considerados como una creación cultural, no como algo natural. Las leyes devienen instrumentos culturales de mediación que posibilitan el desarrollo de acciones histórica y socialmente situadas. Según lo antes explicitado podemos preguntarnos: ¿Cómo las decisiones políticas, intelectuales pueden influir en la realidad de los sujetos en la vida cotidiana?

En la historia de la humanidad, los tratados relativos al matrimonio han sido muy numerosos. En Grecia ${ }^{7}$ se presenta una concepción de la relación matrimonial donde a los principios tradicionales de la complementariedad de los dos sexos necesarios para el orden doméstico, se agrega el ideal de una relación de pareja que compromete todos los aspectos de la vida de los cónyuges y establece vínculos afectivos personales. En esta relación los actos sexuales deben encontrar sus lugares exclusivos, condenando al adulterio tanto como agravio a los privilegios del marido como una agresión al lazo conyugal. Los actos sexuales están ordenados a la procreación, fin que la naturaleza hace recaer en el matrimonio. Los conyugues deben obedecer a una regulación interna exigida por el pudor, la ternura recíproca, el respeto por el otro.

Para Foucault (2001) hay una relación estrecha entre sexualidad, poder y prácticas discursivas. Como tal la monogamia y el matrimonio se constituyen en construcciones de control social en el seno de la burguesía moderna, presentes en las diferentes formas de discurso social.

En cuanto a la homosexualidad, su historia se remonta a la historia de la humanidad. Las formas de legitimación de las relaciones homosexuales han variado en el tiempo, siendo objeto de persecución en diferentes culturas y momentos históricos.

Mediante la enunciación de los derechos y deberes maritales, se logró la objetivación en la institución del matrimonio, de una cierta forma de subjetividad social, que en particular respondía a los espacios de poder de un momento histórico determinado. La institucionalización y legitimación de un arbitrario cultural es el resultado del proceso donde los mecanismos de poder juegan un rol preponderante.

Vemos entonces esta relación entre poder y sexo, que a su vez se traduce en saber, ya que la elaboración de un cierto arbitrario cultural es utilizada por diferentes espacios sociales para la construcción de un discurso. Discurso que forma parte de prácticas cotidianas que identifican a dichos espacios sociales o campos de poder.

\footnotetext{
${ }^{7}$ Musonius Rufus, Antipater de Tarso o Hierocles, Plutarco

RPI Revista de Pesquisa Interdisciplinar, Cajazeiras, v. 1, Ed. Especial, 30-40, set/dez. de 2016.
} 
La ciencia, la pedagogía, la religión, el derecho, los organismos de control (policía) definen unas prácticas discursivas que dan identidad a los sujetos que forman parte de estos campos. Desde la ciencia aparecen diferentes posicionamientos que legitiman la comprensión de la sexualidad en el seno matrimonial, ubicando a toda expresión sexual que estuviera fuera de este marco como desviada. Desde la consideración del pecado y del sacramento (religión), la higiene familiar (medicina), la patologización (psicología y psiquiatría), la judicialización (derecho) y la penalización (policía), la sexualidad fue objetivada y tipificada.

En esta tipificación las relaciones homosexuales entraban en el campo del desvío, la enfermedad, la degeneración, la condena. Pareciera como si todo aquello saliera de la norma, del dogma, fuera considerado una herejía. Herejía que contaba con el apoyo de la religión, la ciencia, la justicia, la economía, la policía y la política.

Actualmente hablamos en Argentina y en muchos lugares del mundo de matrimonio igualitario, podríamos decir que un cierto arbitrario cultural necesita adquirir una institucionalización y legitimación, considerando al proceso de legitimación como "una objetivación de significados de segundo orden (...) La función de la legitimación consiste en lograr que las objetivaciones de primer orden ya institucionalizadas lleguen a ser objetivamente disponibles y subjetivamente plausibles. “ (Berger y Luckmann, 1968: 120)

Tanto desde ciertos sectores sociales como desde la política, se ha apoyado la iniciativa de iguales nombres iguales derechos, ahora bien, ¿qué pasa con los sujetos que hasta hace seis años atrás formaban parte de una sociedad "menos justa", ¿qué pasa con sus posicionamientos frente al matrimonio igualitario',¿ qué voces sustentan sus posiciones?

Aparece entonces un terreno de posicionamiento que da cuenta de trazas, ligadas fuertemente a la identidad homosexual, y a los actos de significado que sustentan a esa identidad.

En la historia de la sexualidad de Foucault aparecen por lo menos tres posicionamientos que desde su origen, han tipificado a las acciones sexuales: unos argumentos de tipo canónicos, relacionados con la génesis, el origen de la vida, las normas de la iglesia católica, etc.; unos argumentos de tipo jurídicos, donde se ha judicializado a las acciones sexuales, ejemplo de ello la penalización de la homosexualidad en muchos países del mundo, la prohibición en la ciudad de Buenos Aires (hasta no hace mucho) que dos hombres estuvieran abrazados, y lo tercero argumento és de tipo patologizante, donde las acciones sexuales consideradas sanas, no patológicas, son aquellas que se enmarcan dentro de lo establecido por el orden social, y toda acción que quede por fuera de este marco, es desviada,

RPI Revista de Pesquisa Interdisciplinar, Cajazeiras, v. 1, Ed. Especial, 30-40, set/dez. de 2016. 
“anormal”, enferma. Es el caso de la concepción de la homosexualidad durante mucho tiempo como un desvío, una enfermedad. Aún hoy, diferentes colectivos sociales en el mundo siguen proponiendo programas de recuperación de los homosexuales.

Finalmente una argumentación de tipo biologicista, ligada a la higiene, a la salud física, a las buenas conductas saludables, donde se estigmatiza y se enmarca a la sexualidad en relación con criterios biológicos.

Al considerar las diferentes opiniones en relación con la ley de matrimonio igualitario, encontramos en las argumentaciones la presencia, la sedimentación de la cultura en trazas del pasado.

En la actualidad, la legalidad y los alcances del matrimonio entre personas del mismo sexo se ha convertido en un tópico presente en los debates sociales en diferentes países. En estos debates aparecen diversos posicionamientos que responden a tradiciones, creencias, valores, representaciones sociales, sobre el matrimonio y la sexualidad.

La ley de matrimonio igualitario en Argentina, ha puesto en evidencia la propuesta del Estado en el desarrollo de acciones de formación cívica que se orientan hacia la construcción de un arbitrario cultural que garantice en una sociedad más inclusiva, la igualdad de derechos a todos los ciudadanos con reconocimiento de la diversidad. Al mismo tiempo nos permite adentrarnos en las particularidades de la educacionalización en la vida cotidiana, considerando la importancia de los medios de comunicación en la difusión, anclaje y objetivación de los objetos culturales.

\section{Interrogantes}

Esta presentación abre el camino hacia diferentes interrogantes en relación con la educacionalización en la vida cotidiana. La centralidad de la sexualidad entorno a la ley de matrimonio igualitario, nos lleva a establecer relaciones entre homosexualidad, matrimonio y derechos civiles.

Sexualidad y matrimonio dialogan en la historia social y a su vez nos permiten adentrarnos en la génesis de los actos de identificación que posibilitan la institucionalización de los objetos culturales. En este sentido, la consideración de la ley de matrimonio igualitario al adquirir un carácter socialmente institucionalizado y legitimado se objetiva como parte 
integrante del colectivo general, la homosexualidad pasa de un carácter restringido y retraído, a una visibilidad social y cultural. Ahora bien esta objetivación es ante todo un proceso de construcción social, donde las prácticas discursivas institucionalizan y legitiman estas nuevas posiciones mediante un proceso dialógico de aprendizaje en la vida cotidiana. Si consideramos el carácter constitutivo y constituyente de estas prácticas discursivas, la cuestión del matrimonio igualitario deviene implícitamente una cuestión que está presente en la subjetividad social, no en un colectivo en particular (ya que surge de ese colectivo: homosexual) sino en el colectivo en general. En tal caso, el presente trabajo nos permite adentrarnos y preguntarnos sobre las significaciones de la sexualidad entre los argentinos luego de la sanción de la ley de matrimonio igualitario y como tal sus cambios en las prácticas sexuales.

Una ley que logra la objetivación de unas subjetividades de un colectivo particular, se institucionaliza y legitima en un contexto general, estando presente en las prácticas discursivas del colectivo general. Las diferentes manifestaciones de esta objetivación parecen hacerse manifiestas en los medios de comunicación y en las expresiones artísticas.

Llegados a este punto encontramos la relación profunda dialógica y dialéctica entre la producción social, las decisiones del Estado y las decisiones personales, y como tal entre la identidad social, procesos de aprendizaje en la vida cotidiana y actos de identificación personal. En este punto matrimonio, homosexualidad y derechos civiles encuentra su punto de integración.

Una ley logra objetivar necesidades, intereses, deseos de una minoría, siendo portadora de nuevas voces pero al mismo tiempo dialogando y tensionando con otras ya vigentes. El anclaje y objetivación de este arbitrario cultural (el matrimonio igualitario) en el colectivo general, debería llevar a la modificación del sistema donde se integra, ya que ningún sistema permanece estático, su propia dinámica lo lleva a la reconstrucción y a la construcción de nuevas configuraciones. Esta modificación en el sistema lleva a nuevos posicionamientos en los sujetos en forma explícita o implícitamente, configurando nuevos marcos referenciales, nuevas voces que posibilitan toma de decisiones y como tal permiten el desarrolla de ciertas acciones. Esta relación en el self entre el yo y el mi parece muy presente en esta descripción. Hay unas acciones presentes y unas voces que de alguna manera dialogan y tensionan con esas acciones presentes. Finalmente el sujeto lleva adelante sus acciones como sujeto sexuado y situado, acciones que devienen prácticas sociales, portadoras de significaciones y sentidos, que irán adquiriendo una tipificación social.

RPI Revista de Pesquisa Interdisciplinar, Cajazeiras, v. 1, Ed. Especial, 30-40, set/dez. de 2016. 
El camino recorrido abre a su vez nuevos interrogantes. Teniendo en cuenta que el matrimonio igualitario al incluir a las minorías homosexuales en la institución matrimonio tipifica a estos nuevos actores poniéndolos en igualdad de condiciones respecto de los heterosexuales, esta modificación, transformación, cambio, en la objetivación matrimonio ¿Cómo influyó en la construcción de las subjetividades de los actores heterosexuales en relación con su sexualidad, el matrimonio, y derechos civiles?.

\section{BIBLIOGRAFÍA}

BAJTÍN, M. Estética de la creación verbal. México, Siglo XXI, 1991.

BAJTIN, M; VOLOSHINOV, V: ( ) El marxismo y la filosofía del lenguaje: Los principales problemas del método sociológico en la ciencia del lenguaje. (Trad. Tatiana Bubnova) Alianza Editorial. 1era Edición. Madrid, 1992

BERGER, P y LUCKMAN, Th. La construcción social de la realidad. Buenos Aires. Amorrortu Editores, 1968.

BOURDIEU, P. Razones prácticas. Barcelona: Anagrama, 1997.

BOURDIEU, P. y Passeron, J. C.La reproducción. Elementos para una teoría del sistema de enseñanza”. Barcelona: Laia, 1977.

DEL RÍO, P. y ALVAREZ, A. La educación como construcción cultural en un mundo cambiante. En A, Álvarez; P, Del Rio y J, Wertsch, (comp.), Explorations in SocioCultural Studies (pp 239-253) (vol.4). Madrid: Fundación Infancia y Aprendizaje, 1994 FOUCAULT, M.Historia de la sexualidad. Buenos Aires. Siglo XXI Editores Argentina, 2002.

LAVE, J. La cognición en la práctica. Buenos Aires, Paidós, 1991.

ROGOFF, B. Los tres planos de la actividad socio-cultural: apropiación participativa, participación guiada y aprendizaje" en Wertsch, J. ; del Río, P. y Alvarez, A. (Eds.) La mente sociocultural. Aproximaciones teóricas y aplicadas, Madrid: Fundación Infancia y Aprendizaje, 1997.

WERTSCH, J. Un enfoque socio-cultural de la accion mental. En Carretero, M. (comp.) Desarrollo y aprendizaje. Buenos Aires, Aique, 1998.

Voces de la mente Madrid, Ed. Visor Distribuciones, 1993.

La mente en acción. Buenos Aires. Aique, 1999

RPI Revista de Pesquisa Interdisciplinar, Cajazeiras, v. 1, Ed. Especial, 30-40, set/dez. de 2016. 